controls. Sound sequences containing duration, intensity and frequency deviant sounds at low $(6 \%)$ or high $(20 \%)$ probability were presented while participants viewed a movie with low-level soundtrack.

Results: A repeated-measures ANOVA was used to compare MMN amplitude across groups (patients/ controls) and deviant type (duration/frequency/intensity) and probability (low/high) with age as a covariate. There were significant main effects of probability and deviant type both modified by age and a main effect of group modified by probability. Patients produced significantly smaller MMN responses to low- but not high-probability deviants. Age was a significant covariate in the low-probability but not the high-probability condition with the differences being more pronounced for frequency and intensity MMN than duration MMN.

Conclusions: MMN amplitude was significantly reduced in schizophrenia vs. controls for the lowprobability condition only (ie when under conditions of increased repetition of the standard sound). The agerelated decline in MMN was also most pronounced under these conditions. The results are discussed with respect to current research into memorybased and discrimination-based conceptualizations of the MMN.

\section{Depression and anxiety in cardiac rehabilitation patients: characteristics, treatment and outcome}

\section{A Turner', J Hambridge², A Baker', F Kay-Lambkin', L Phillips', J Bowman'}

${ }^{1}$ Centre for Mental Health Studies, The University of Newcastle; and ${ }^{2} J o h n$ Hunter Hospital, Hunter New England Health, Newcastle, Australia

Background: The past decade has seen a growing body of evidence to support independent links between depression and coronary heart disease. Despite this evidence, depression is rarely assessed in cardiac rehabilitation programs and there are few published studies of psychological interventions for depression with this population. The aim of the present evaluation was to first determine levels of depression and anxiety symptoms among cardiac rehabilitation patients in John Hunter Hospital (JHH), Newcastle, and second to link those scores with demographic, lifestyle and medical variables. Additionally, it was aimed to evaluate a group cognitive behaviour therapy intervention (BraveHeart), specialized for treatment of depression in people with cardiac disease.

Methods and Results: The Hospital Anxiety and Depression Scale is being used to screen cardiac rehabilitation patients at $\mathrm{JHH}$ at week 4 of their program. Over 650 patients have been screened with this tool, with results suggesting that around a third are experiencing significant levels of anxiety and/ or depression. These scores have been linked with available patient information kept on an epidemiological database, the Heart and Stroke Register, to determine the characteristics and medical outcome of those with high vs. low symptom scores. A randomized controlled trial of BraveHeart has commenced and preliminary data from the study will be presented.

Conclusions: Results from screening support prior research suggesting that significant levels of emotional distress exist among cardiac rehabilitation participants. Depression is known to lead to worse outcomes among this patient population, and development of efficacious psychological treatments is indicated.

\section{Differential effects of antipsychotic drugs on serotonin- $1 \mathrm{~A}$ receptor- mediated disruption of prepulse inhibition}

\section{$M$ van den Buuse}

Behavioural Neuroscience Laboratory, Mental Health Research Institute of Victoria, Melbourne, Australia

Background and Methods: Serotonin-1A (5-HT1A) receptors have been implicated in the symptoms of schizophrenia. However, there is limited in vivo evidence for an interaction of antipsychotic drugs with 5-HT1A receptor-mediated behavioural effects. We therefore investigated in rats the action of several antipsychotic drugs on prepulse inhibition (PPI), a measure of sensorimotor gating, which is deficient in schizophrenia. Disruption of PPI was induced by treatment with $0.5 \mathrm{mg} / \mathrm{kg}$ of the 5-HT1A receptor agonist, 8-hydroxy-di-propyl-aminotetralin (8-OH-DPAT).

Results: In rats pretreated with $0.25 \mathrm{mg} / \mathrm{kg}$ of haloperidol or raclopride, the disruption of PPI was no longer significant. Of the atypical antipsychotic drugs clozapine, olanzapine, risperidone, amisulpride and aripiprazole, only aripiprazole significantly reduced the effect of 8-OH-DPAT on PPI. This effect was mimicked by pretreatment with the 5-HT1A receptor partial agonist, buspirone. On the other hand, some of the antipsychotic drugs and other pretreatments showed complex, prepulse-dependent effects on their own, both on PPI and prepulse facilitation at the $30 \mathrm{~ms}$ ISI (clozapine, risperidone, amisulpride) and PPI at the $100 \mathrm{~ms}$ ISI (olanzapine, risperidone, MDL 73,005EF).

Conclusions: These data show little in vivo interaction of several atypical antipsychotic drugs with the 